\title{
Cherenkov Telescope Array sensitivity to branon dark matter models
}

\author{
A. Aguirre-Santaella, ${ }^{a, b, *}$ V. Gammaldi, ${ }^{a, b}$ M. A. Sánchez-Conde ${ }^{a, b}$ and D. Nieto ${ }^{c}$ \\ ${ }^{a}$ Instituto de Física Teórica UAM-CSIC, Universidad Autónoma de Madrid, \\ C/ Nicolás Cabrera, 13-15, 28049 Madrid, Spain \\ ${ }^{b}$ Departamento de Física Teórica, M-15, Universidad Autónoma de Madrid, \\ E-28049 Madrid, Spain \\ ${ }^{c}$ Grupo de Altas Energías and IPARCOS, Universidad Complutense de Madrid, \\ Av Complutense s/n, 28040 Madrid, Spain \\ E-mail: alejandra.aguirre@uam.es, viviana.gammaldi@uam.es, \\ miguel.sanchezconde@uam.es, d.nieto@ucm.es
}

TeV DM candidates are gradually earning more and more attention within the community. Among others, extra-dimensional brane-world models may produce thermal DM candidates with masses up to $100 \mathrm{TeV}$, which could be detected with the next generation of very-high-energy gamma-ray observatories such as the Cherenkov Telescope Array (CTA). In this work, we study the sensitivity of CTA to branon DM via the observation of dwarf spheroidal galaxies. We computed annihilation cross section values needed to reach a $5 \sigma$ detection as a function of the branon mass. Additionally, in the absence of a predicted DM signal, we obtained $2 \sigma$ upper limits on the annihilation cross section. These limits lie $1.5-2$ orders of magnitude above the thermal relic cross section value. Yet, CTA will allow to exclude a significant portion of the brane tension-mass parameter space in the $0.1-60 \mathrm{TeV}$ branon mass range, and up to tensions of $\sim 10 \mathrm{TeV}$. More importantly, CTA will significantly enlarge the region already excluded by AMS and CMS, and will provide valuable complementary information to future SKA radio observations.

Based on JCAP 10 (2020) 041, arXiv:2006.16706 [1]

$37^{\text {th }}$ International Cosmic Ray Conference (ICRC 2021)

July 12th - 23rd, 2021

Online - Berlin, Germany

\footnotetext{
${ }^{*}$ Presenter
} 


\section{Introduction}

Gamma-ray astronomy represents a unique window for the indirect detection of weakly interacting massive particles (WIMPs) with masses in the range from $\mathrm{MeV}$ to $\mathrm{TeV}$. In the near future, new gamma-ray observatories will surely continue the search. Among others, the Cherenkov Telescope Array $(\mathrm{CTA})^{1}$ [2], an international project that will build the next generation ground-based gamma-ray observatory, will be well positioned to shed further light on the nature of dark matter (DM).

In this work, we will study CTA sensitivity to a particular type of WIMPs, i.e., branons [3]. Branons generate from the effective theory of extra-dimensional brane-world and may offer thermal DM candidates with masses up to $100 \mathrm{TeV}$; thus they represent good DM candidates for CTA, which is useful for TeV DM searches, due to an energy range that covers from $20 \mathrm{GeV}$ to $300 \mathrm{TeV}$. In the past, a thermal branon with mass of $\sim 50 \mathrm{TeV}$ was proposed to explain the cut-off at $\mathrm{TeV}$ scale observed by the H.E.S.S. telescope at the Galactic center [4].

\section{Gamma-ray fluxes from branon annihilation}

Branons are WIMPs that represent a viable candidate for DM, and may then annihilate into e.g. a pair of quarks, a pair of weak bosons, or even a pair of photons, yet the probability for the latter to occur is especially low [3]. The branching ratio of annihilation into each Standard Model (SM) channel depends on the mass of the branons $\left(m_{\chi}\right)$ and a parameter related to the brane tension, $f$. In case branons are considered thermal relics and their annihilation cross-section value is the one needed to account for $100 \%$ of the total DM content of the Universe, the tension is a function of the branon mass, and we just have one free parameter. This is shown in the left panel of figure 1 . The right panel of the same figure contains the branching ratios for different branon masses. It can be seen that, in our relevant energy band, if $m_{\chi}$ is below the kinematic threshold for $W^{+} W^{-}$production, the $b \bar{b}$ channel is the leading one; otherwise, the $W^{+} W^{-}$channel dominates.

Since we need two DM particles to have an annihilation, and each annihilation can produce several photons, the number of potential encounters within DM particles will be proportional to the DM number density, $n_{\chi}$, squared, and the number of generated photons will be proportional to $n_{\chi}^{2}$ times the velocity-averaged annihilation cross section, $\langle\sigma v\rangle$, which represents the interaction likelihood. With this in mind, the differential annihilation flux will thus be

$$
\frac{d \phi}{d E}=\int_{\text {l.o.s. }} \rho^{2}(r) d r \frac{\langle\sigma v\rangle}{2 \pi m_{\chi}^{2}} \sum_{i} B r_{i} \frac{d N_{i}}{d E}
$$

Note that the astrophysical factor, $J=\int_{\text {l.o.s. }} \rho^{2}(r) d r$, takes into account the integration along the line of sight (1.o.s.) of the DM density distribution $\rho(r)$ in the source squared. In our work, we will not include J-factor uncertainties and we will simply consider benchmark values in section 3 . Instead, we will pay particular attention to the particle physics factor, i.e. the factor related to the photon yield. Indeed, $\langle\sigma v\rangle_{\mathrm{th}}=3 \times 10^{-26} \mathrm{~cm}^{3} \mathrm{~s}^{-1}$ is the thermally-averaged cross section of the DM particle times the velocity, and $B r_{i}=\langle\sigma v\rangle_{\mathrm{i}} /\langle\sigma v\rangle$ is the branching ratio of annihilation channel $i$.

\footnotetext{
1https://wWw.cta-observatory.org/
} 

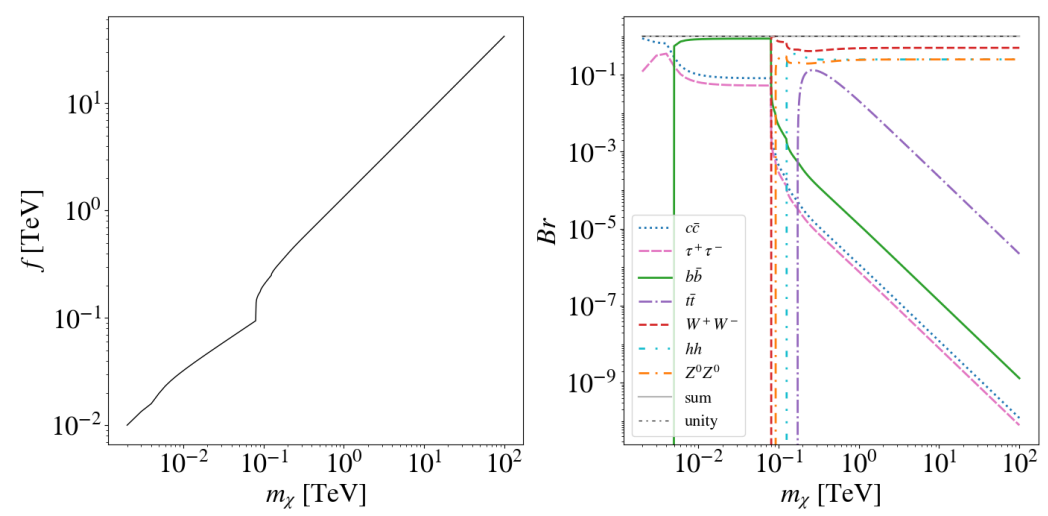

Figure 1: Left: tension of the brane $(f)$ as a function of the DM (branon) mass $\left(m_{\chi}\right)$ when the branon is considered to be a thermal relic and can account for the total DM content of the Universe. Right: Branching ratios of the most relevant annihilation channels, defined according to eq. 1, for branons with a mass ranging from a few $\mathrm{GeV}$ to $100 \mathrm{TeV}$. The sum of all branching ratios at a given mass is the unity. Expressions for $f$ and branching ratios taken from [5]. Figure taken from [1].

With the branon branching ratios of figure 1, the expected gamma-ray flux from the annihilation of branon DM has a well-defined spectrum, depending on the mass of the particle itself, as shown in figure 2. The spectra using the normalized energy have roughly the same shape, especially at intermediate values of the dimensionless parameter $x=2 E_{\gamma} / E_{\mathrm{CM}}$, where $E_{\gamma}$ is the energy of the emitted gamma-ray photons and $E_{\mathrm{CM}}$ is the energy of the center of mass of the simulated event. This variable is reduced to $x=E_{\gamma} / m_{\chi}$ in the case of annihilating DM.
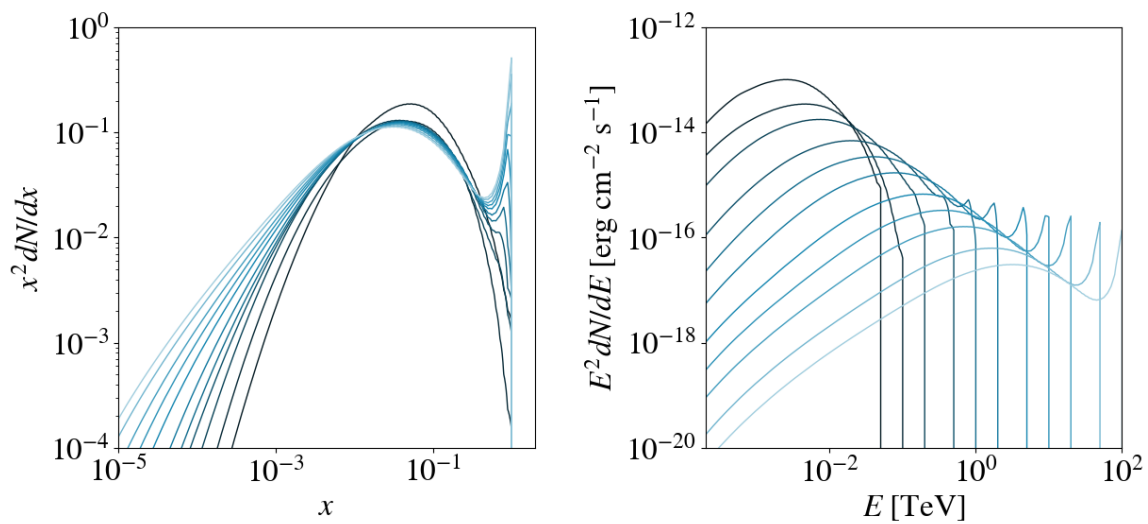

Figure 2: Annihilation spectra for different branon masses, from dark to light blue: $0.05,0.1,0.2,0.5,1$, 2, 5, 10, 20, 50 and $100 \mathrm{TeV}$. Left: photon yield in terms of the energy normalized to the branon mass, $x=E_{\gamma} / m_{\chi}$, times this normalized energy squared. Right: differential flux times the energy squared in terms of the energy for the example case of adopting $J=1.42 \cdot 10^{19} \mathrm{GeV}^{2} \mathrm{~cm}^{-5}$ (similar to Draco's; see section 3). Photon fluxes obtained with the tools in [6, 7]. Figure taken from [1]. 


\subsection{The Cherenkov Telescope Array}

CTA is an international project to build the next generation ground-based gamma-ray observatory, that will be sensitive to gamma rays in the energy range from $20 \mathrm{GeV}$ to $300 \mathrm{TeV}$ [2]. CTA is designed as an open observatory and will consist of two arrays of imaging atmospheric Cherenkov telescopes (IACTs), one at the Northern Hemisphere (La Palma, Canary Islands, Spain), devoted to the study of extragalactic objects down to the lowest CTA achievable energies, and another array at the Southern Hemisphere (Atacama Desert, Chile), that will mostly concentrate on Galactic sources.

\subsection{Analysis pipeline}

In this section, we will depict the simulations of the detection and data analysis procedure of the observations of Draco and Sculptor by the CTA Observatory.

In particular, we have used the ctools software package [8] to both generate the simulations and run the successive data analyses. ctools was developed to offer easy software tools that could be used to analyze high-level data collected by IACTs. The software operates with reconstructed event lists. The user feeds ctools with the instrument response functions (IRFs), that describe the transformation between physical photon features and measured event features. IRFs also add background, i.e., events that look like photons to the analysis but are actually cosmic rays (electrons and hadrons). In our case, we have made use of the (prod3b-v2) version of the publicly available CTA IRFs $^{2}$. The main IRFs are the effective area, the energy resolution, the angular resolution and the background rate.

A flowchart that describes the whole simulation chain is shown in figure 3 .

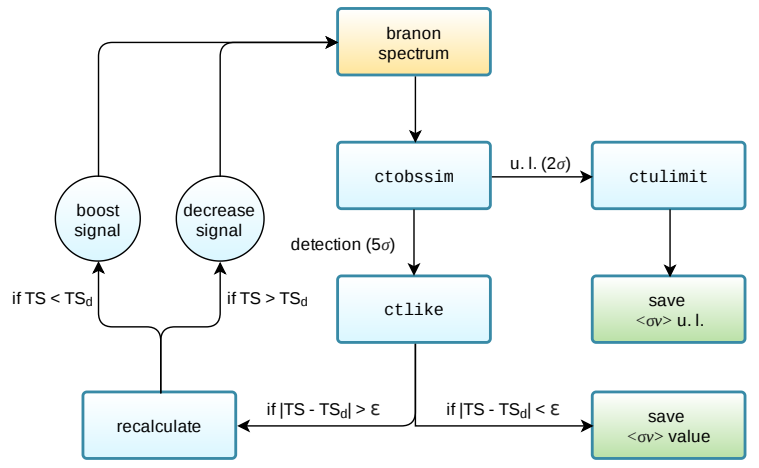

Figure 3: Flowchart of the simulation chain, both for the case of studying branon detection and for setting upper limits on the annihilation cross section. Figure taken from [1].

\section{Detection prospects and constraints on branon DM models}

\subsection{Targets}

We have focused on two dSphs for our analysis, Draco and Sculptor, as representative cases of the so-called "classical" dwarfs. The chosen dSph parameters are shown in table 1. Note that

\footnotetext{
2https://wwW.cta-observatory.org/science/cta-performance/
} 
we selected one dSph in each hemisphere, so as to perform a study of the sensitivities of both CTA-South and CTA-North to branons. Both dSphs are assumed to be point-like, which is a fair approximation given the angular size subtended by the bulk of the DM-induced emission in these objects and the CTA point spread function.

For each selected target we simulate CTA observations with total exposures of $300 \mathrm{~h}$ per dSph. We expect this amount of exposure time to be dedicated to observations of dSphs in the first 3 years of scientific operation of CTA [2].

\begin{tabular}{|cccccc|}
\hline Target & $m_{\chi}[\mathrm{TeV}]$ & $T_{o b s}[\mathrm{~h}]$ & $(\mathrm{RA}, \mathrm{DEC})[\mathrm{deg}]$ & $J\left[\frac{\mathrm{GeV}^{2}}{\mathrm{~cm}^{5}}\right]$ & $\left(E_{\min }, E_{\max }\right)[\mathrm{TeV}]$ \\
\hline Draco & {$[0.1,60]$} & 300 & $(260.05,57.915)$ & $1.42 \cdot 10^{19}$ & $\left(0.03, m_{\chi}\right)$ \\
Sculptor & {$[0.1,60]$} & 300 & $(15.0375,-33.7092)$ & $3.56 \cdot 10^{18}$ & $\left(0.03, m_{\chi}\right)$ \\
\hline
\end{tabular}

Table 1: Targets used in this analysis and most relevant parameters for the CTA simulations. Sources are assumed to be point-like. Right ascension (RA) and declination (DEC) refer to the target coordinates, which are slightly different from the center of the CTA field of view (we use an offset of 0.5 deg for the observations). J-factors obtained from [9].

\subsection{Annihilation cross section values}

Here, we provide the results we obtained in our analysis of CTA simulation data using the parameters in table 1 . The annihilation cross section values that would be needed for detection (left panels) and those corresponding to $95 \%$ c.l. upper limits in the case of no detection (right panels) are illustrated in figures 4 and 5 for Draco and Sculptor, respectively [1]. From the left panels, one can derive that it is very unlikely that CTA could detect thermal branons in these objects. Actually, if an hypothetical detection occurred, we would be two orders of magnitude further away from the thermal relic cross section. A similar situation can be seen in the right panels of figures 4 and 5: CTA will not be able to reject any mass for thermal branons, since the exclusion limits lie around 1.5 orders of magnitude, at best, above the thermal cross section value.

In the right panel of figure 5, we also compare our attained upper limits with results shown in [10] for Sculptor and the $W^{+} W^{-}$channel.

In the case the branon is assumed to be a thermal relic, we note that the tension of the brane is completely determined by the mass of the branon (figure 1, left panel). In this scenario, it is then possible to convert limits to the annihilation cross section as a function of the branon mass into limits to the branon tension as a function of the branon mass. To do so, we translate the obtained 95\% upper limits on the annihilation cross section into $2 \sigma$ exclusion regions in the tension-mass parameter space first introduced in figure 1 in the following way [1,3]:

$$
f=\left(\frac{\hbar^{2} c^{3}}{\langle\sigma v\rangle} \sum_{i} d_{0, i}\right)^{1 / 8}
$$

where we sum over all annihilation channels $i, \hbar$ is the reduced Planck constant, $c$ is the speed of light, and the channel-dependent constants, $d_{0, i}$, are proportional to the leading order component of the annihilation cross section into channel $i$. 

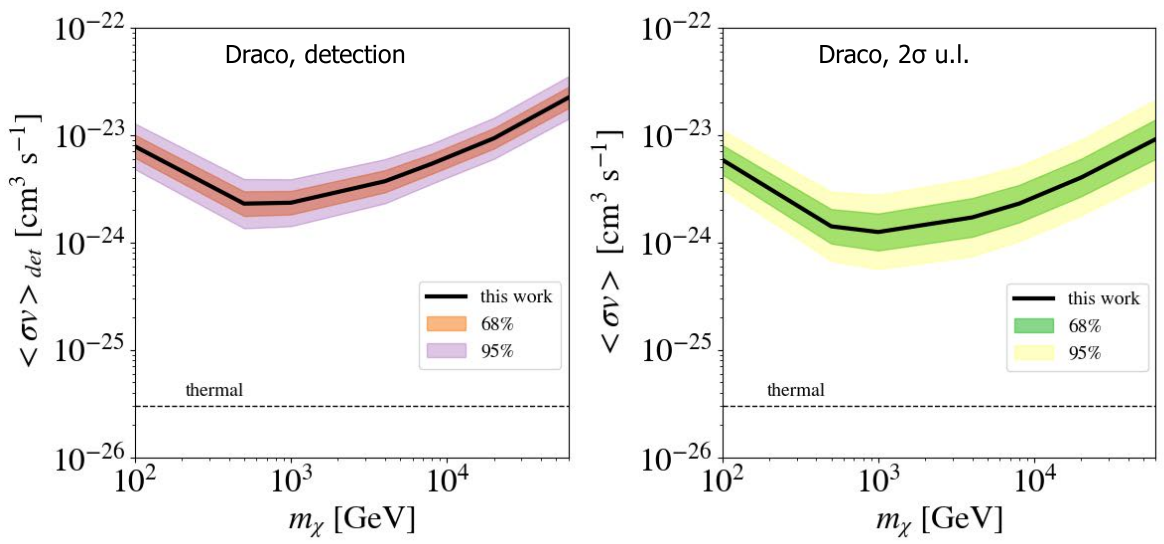

Figure 4: Annihilation cross section values, as a function of the branon mass, needed for a $5 \sigma$ detection with CTA (left) and for the case of setting $2 \sigma$ upper limits in the absence of a branon signal (right). These results refer to $300 \mathrm{~h}$ of observation time of the Draco dSph by CTA-North, adopting the parameters detailed in table 1. Coloured areas correspond to $68 \%$ and $95 \%$ confidence bands as obtained from 100 independent observation simulations. The horizontal dashed line corresponds to the thermal value of the annihilation cross section. Figure taken from [1].
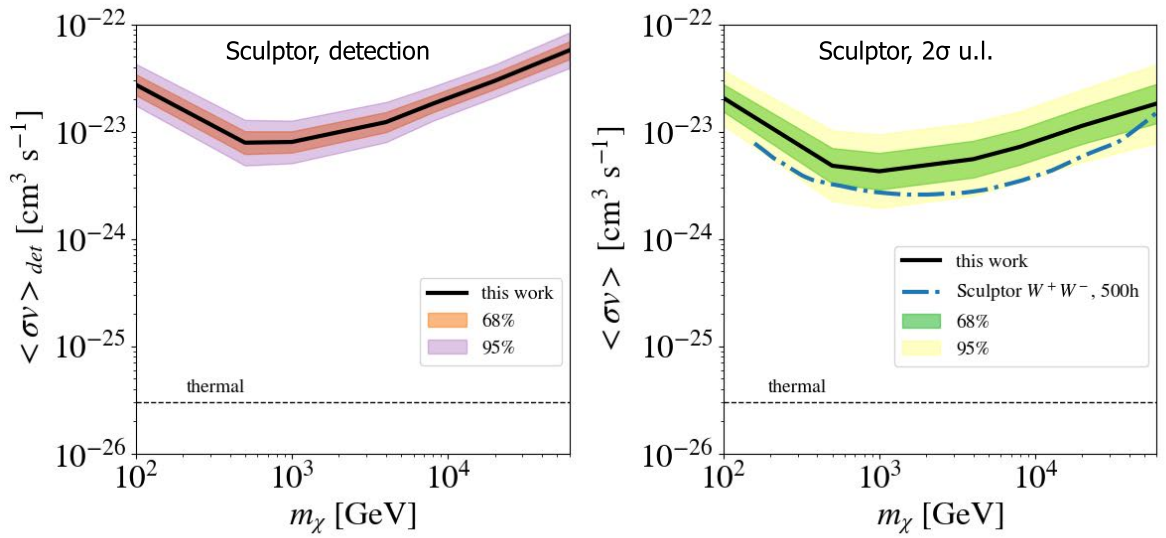

Figure 5: Annihilation cross section values, as a function of the branon mass, needed for a $5 \sigma$ detection with CTA (left) and for the case of setting $2 \sigma$ upper limits in the absence of a branon signal (right). These results refer to $300 \mathrm{~h}$ of observation time of the Sculptor dSph by CTA-South, adopting the parameters detailed in table 1. Coloured areas correspond to $68 \%$ and $95 \%$ confidence bands as obtained from 100 independent observation simulations. The horizontal dashed line corresponds to the thermal value of the annihilation cross section. In the right panel, we also show the upper limits predicted for this same object (dash-dotted blue line) in case of WIMPs annihilating to $W^{+} W^{-}$and 500 hours of observation taken from [10]. Figure taken from [1].

The result of this study is depicted in figure 6, where it is shown that CTA will exclude a large region of the mass-tension parameter space, $f\left(m_{\chi}\right)$, enclosing approximately mass values between 
0.1 and $60 \mathrm{TeV}$, and tension values up to $10 \mathrm{TeV}$ for the largest masses. In the same figure, we also compare with results obtained from the Alpha Magnetic Spectrometer, AMS, and the Compact Muon Solenoid, CMS, as well as prospects for the future Square Kilometre Array, SKA. As it can be seen, all of these exclusion regions are nicely complementary, our results expanding the excluded region for $\mathrm{TeV}$ masses with respect to the limits from current experiments.

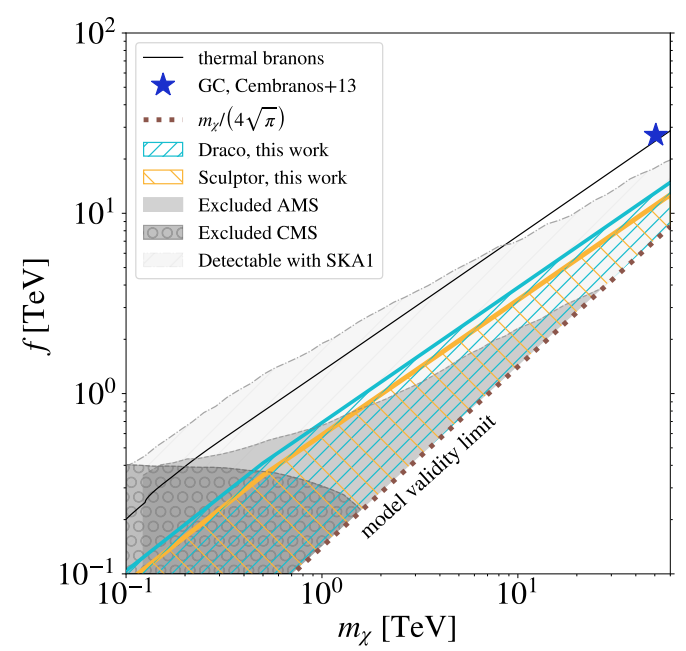

Figure 6: Branon tension as a function of its mass. The thick dotted brown line at the very bottom shows the limit of validity for the model in the $f\left(m_{\chi}\right)$ parameter space. The black line -already shown in figure 1shows the relation between tension and branon mass if these were thermal relics. Orange and blue areas correspond to regions of the parameter space that would be excluded at $2 \sigma$ c.l. by the upper limits obtained in the right panels of figures 4 and 5 for Draco and Sculptor, respectively. Dark gray regions are those that have been already excluded by CMS and AMS, while the light gray region refers to prospects for SKA from synchrotron radio emission expected from DM annihilation in Draco. The dark blue star refers to the branon model that would provide an alternative explanation to the observation of very high energy gamma-rays at the Galactic center (GC) with HESS. Figure taken from [1].

\section{Discussion and conclusions}

In this work, we have studied the sensitivity of the future Cherenkov Telescope Array to branons. In the limit of thermal branons, the branching ratios into different annihilation channels depend only on the mass of the particle.

We focus on two of the best astrophysical targets traditionally identified in gamma-ray DM searches, namely the Draco and Sculptor dSphs, located in the Northern and Southern Hemispheres, respectively. We explored two scenarios in our analysis: detection and upper limits to the annihilation cross section in case of no signal. In the former, we computed the required averaged annihilation cross sections required for a CTA detection, while in the latter we first obtained the corresponding $95 \%$ c.l. upper limits to the annihilation flux for different branon masses, and then translated this information into $95 \%$ c.l. upper limits to the annihilation cross section as well.

Though CTA will not be able to test thermal branons by means of Draco and Sculptor observations, our results being $1.5-2$ orders of magnitude above the thermal relic cross section value, 
CTA observations will allow us to exclude a significant portion of the brane tension versus branon mass parameter space, $f\left(m_{\chi}\right)$, ranging from 0.1 to $60 \mathrm{TeV}$ in branon mass and up to $\sim 10 \mathrm{TeV}$ of the brane tension, as shown in figure 6. In a more general context, we note that CTA will be enlarging the region of the parameter space that has already been excluded by AMS and CMS by other means, especially for branon masses above $1 \mathrm{TeV}$. CTA will also probe a large fraction of the exclusion region achievable by SKA using radio observations, this way providing valuable complementary information.

As a general remark from this first work on CTA and branons, we conclude that CTA possess a superb potential to pursue this kind of DM searches and, in particular, to test well-motivated TeV DM candidates like the one here discussed. In this enterprise, CTA will also offer the necessary complementarity with other current and future instruments that may test branon models by means of different messengers, approaches or frequencies, such as AMS, CMS and SKA.

\section{References}

[1] A. Aguirre-Santaella, V. Gammaldi, M.A. Sánchez-Conde and D. Nieto, Cherenkov Telescope Array sensitivity to branon dark matter models, JCAP 2020 (2020) 041 [2006.16706].

[2] CTA Consortium collaboration, Science with the Cherenkov Telescope Array, WSP (2019) [1709.07997].

[3] J.A.R. Cembranos, A. Dobado and A.L. Maroto, Brane world dark matter, Phys. Rev. Lett. 90 (2003) 241301 [hep-ph/0302041].

[4] V. Gammaldi, Multimessenger Multi-TeV Dark Matter, Front. Astron. Space Sci. 6 (2019) 19 [1903.05010].

[5] J.A.R. Cembranos, A. Dobado and A.L. Maroto, Cosmological and astrophysical limits on brane fluctuations, Phys. Rev. D 68 (2003) 103505 [hep-ph/0307062].

[6] M. Cirelli et al., PPPC 4 DM ID: A Poor Particle Physicist Cookbook for Dark Matter Indirect Detection, JCAP 03 (2011) 051 [1012 . 4515].

[7] P. Ciafaloni, D. Comelli, A. Riotto, F. Sala, A. Strumia and A. Urbano, Weak Corrections are Relevant for Dark Matter Indirect Detection, JCAP 03 (2011) 019 [1009. 0224].

[8] J. Knödlseder et al., GammaLib and ctools. A software framework for the analysis of astronomical gamma-ray data, Astron. Astrophys. 593 (2016) A1 [1606.00393].

[9] V. Bonnivard et al., Dark matter annihilation and decay in dwarf spheroidal galaxies: The classical and ultrafaint dSphs, Mon. Not. Roy. Astron. Soc. 453 (2015) 849 [1504 . 02048].

[10] CTA collaboration, Prospects for Indirect Dark Matter Searches with the Cherenkov Telescope Array (CTA), PoS ICRC2015 (2016) 1203 [1508. 06128]. 